\title{
RADIAL DISTRIBUTIONS OF MAGNETIC FIELD STRENGTH IN THE SOLAR CORONA AS DERIVED FROM DATA ON FAST HALO CMES
}

\author{
V.G. Fainshtein \\ Institute of Solar-Terrestrial Physics SB RAS, \\ Irkutsk, Russia,vfain@iszf.irk.ru
}

\author{
Ya.I. Egorov \\ Institute of Solar-Terrestrial Physics SB RAS, \\ Irkutsk,Russia,egorov@iszf.irk.ru
}

\begin{abstract}
In recent years, information about the distance between the body of rapid coronal mass ejection (CME) and the associated shock wave has been used to measure the magnetic field in the solar corona. In all cases, this technique allows us to find coronal magnetic field radial profiles $B(R)$ applied to the directions almost perpendicular to the line of sight. We have determined radial distributions of magnetic field strength along the directions close to the Sun-Earth axis. For this purpose, using the "ice-cream cone" model and SOHO/LASCO data, we found $3 \mathrm{D}$ characteristics for fast halo coronal mass ejections (HCMEs) and for HCME-related shocks. With these data we managed to obtain the $B(R)$ distributions as far as $\approx 43$ solar radii from the Sun's center, which is approximately twice as far as those in other studies based on LASCO data. We have concluded that
\end{abstract}

to improve the accuracy of this method for finding the coronal magnetic field we should develop a technique for detecting CME parts moving in the slow and fast solar wind. We propose a technique for selecting CMEs whose central (paraxial) part actually moves in the slow wind.

Keywords: CME, shock, solar wind, magnetic field.

\section{INTRODUCTION}

Coronal plasma is immersed in the non-uniform and anisotropic magnetic field. The solar corona structure and its associated explosive, eruptive processes are closely related to characteristics of the magnetic field at different spatial scales. Thereby, developing reliable and precise methods for determining the coronal magnetic field is one of the key problems solar scientists face.

There have been no regular measurements of the coronal magnetic field - only individual ones based on the Zeeman effect (in the infrared band) [Lin et al., 2000], Hanle effect [Sahal-Brechot et al., 1986], and Faraday effect [Pätzold et al., 1987; Spangler, 2005], as well as on measurements of solar radio emission [Lee, 2007; Bogod, Yasnov, 2016]. The coronal magnetic field can be reconstructed in different approximations by measuring the photospheric field: in the potential approximation (see [Rudenko, 2001] and references therein), in the force-free approximation [Wiegelmann, 2008; Rudenko, Myshyakov, 2009]. Some estimates of the coronal magnetic field were obtained taking into account the relationship of the field with different independently determined characteristics of the coronal plasma, such as the $\beta$ parameter, gyrofrequency, Alfvén velocity (see the monograph by Schwenn, Marsch [1990] and references therein).

In [Gopalswamy, Yashiro, 2011; Kim et al., 2012], a new method for finding coronal magnetic field values has been proposed and tested. The method relies on the existence of the relationship of the normalized standoff distance from the CME part (CME "nose”) most distant from the Sun to the CME associated shock with the Alfvén Mach number $M$ [Russell, Mulligan, 2002].], and hence with the Alfvén velocity. To find $M$, Kim et al. [2012] also used the relationship between the Mach number and the density jump in the shock front [Landau, Lifshitz, 1988]. The method [Gopalswamy, Yashiro, 2011; Kim et al., 2012] was applied to find magnetic field radial distributions $B(R)$ in the plane of the sky approximately up to $20 R_{\mathrm{S}}\left(R_{\mathrm{S}}\right.$ is the solar radius) from COR2, C2, and C3 coronagraph data. The COR2 coronagraphs are part of the Sun Earth Connection Coronal and Heliospheric Investigation (SECCHI) instrument suite [Howard et al., 2008] on the Solar Terrestrial Relations Observatory (STEREO) mission [Kaiser et al., 2008]; the C2 and C3 coronagraphs are part of the Large Angle and Spectrometric Coronagraph (LASCO) [Brueckner et al., 1995] on the Solar and Heliospheric Observatory (SOHO) mission. In this case, $B(R)$ was found by analyzing limb CMEs, i.e. mass ejections whose sources are relatively close to the solar limb. Poomvises et al. [2012] applied this method to distances up to $\approx 120 R_{\mathrm{S}}$, using SOHO/LASCO and Heliospheric Imager 1 data. Heliospheric Imager 1 telescopes are part of the SECCHI suite. Schmidt et al. [2016] for the CME registered on November 29, 2013 have compared radial distributions of the magnetic field, obtained using the method put forward in [Gopalswamy, Yashiro, 2011] and magnetohydrodynamic 3D calculations of the shock-related model CME motion. The authors have concluded that the magnetic field radial distributions obtained by the two methods at $(1.8-10) R_{\mathrm{S}}$ agree well. 
We adopt the method of finding the magnetic induction value in the outer corona, proposed by Gopalswamy and Yashiro [2011], to fast coronal mass ejections of halo type (HCMEs) with sources of most of them located near the center of the solar disk. Such CMEs move at a slight angle to the Sun-Earth axis [Fainshtein, 2006]. Therefore, to find the kinematic characteristics of CME body and shock wave necessary to determine the magnetic field distribution along the direction of such CMEs, we should calculate CME characteristics in 3D space. To determine the position and velocity of the boundary of the HCME body and its related shock in 3D space, we use the ice-cream cone model described in [Xue et al., 2005]. We compare the magnetic field radial distributions obtained using the HCME characteristics with the field distributions derived from the limb CME observations in [Kim et al., 2012]. Finally, the paper discusses the problem of determining the type (fast or slow) of the solar wind (SW) within which a certain part of CME body moves. As a first step in solving this problem, we propose a method for selecting CMEs with their central part moving in the slow SW.

\section{DATA AND RESEARCH TECHNIQUES}

The fast HCMEs selected for the analysis are listed in Table 1. For each event, we give the date and time of the first HCME recording in the LASCO C2 field of view, the linear projection CME velocity, coordinates and X-ray class of a HCME-related flare from the catalog [https://cdaw.gsfc.nasa.gov/CME list/HALO/halo .html].

To find HCME 3D parameters (the position of the HCME axis in space, or, what is the same, HCME direction, the HCME angular size $2 \alpha_{\mathrm{CME}}$, the position $r_{\mathrm{p}}$ and velocity $v_{\mathrm{p}}$ of the HCME outer boundary on the axis of the model as a function of time), we adopt the method proposed by Xue et al. [2005], who used the socalled ice-cream cone model as a CME model.

Table 1

\begin{tabular}{|c|c|c|c|c|}
\hline $\begin{array}{c}\text { Date, day- } \\
\text { month-year }\end{array}$ & Time & Speed, km/s & Flare coordinates & Flare class \\
\hline 18.11 .03 & $8: 50: 05$ & 1660 & N00E18 & M3.9 \\
\hline 06.04 .04 & $13: 31: 43$ & 1368 & S18E15 & M2.4 \\
\hline 03.11 .04 & $16: 54: 05$ & 1759 & N09W17 & X2.0 \\
\hline 07.11 .04 & $16: 54: 05$ & 1759 & N09W17 & X2.0 \\
\hline 15.01 .05 & $23: 06: 50$ & 2861 & N15W05 & X2.6 \\
\hline 17.01 .05 & $9: 30: 05$ & 2094 & N13W19 & X2.2 \\
\hline 30.07 .05 & $6: 50: 28$ & 1968 & N12E60 & X1.3 \\
\hline 05.09 .05 & $19: 48: 05$ & 2257 & source after limb & - \\
\hline 13.09 .05 & $20: 00: 05$ & 1866 & S09E10 & X1.5. \\
\hline
\end{tabular}

This model represents CME as a cone with a top at the Sun's center. The cone leans against a part of the spherical surface with a radius equal to the cone generator length. The HCME direction is determined by the spatial position of the model cone axis, which is described by two angles: $\theta_{0}$ and $\varphi_{0}$. The $\theta_{0}$ angle (colatitude, $\left.\theta_{0}=\left[0^{\circ}-180^{\circ}\right]\right)$ is counted clockwise from the positive direction of the axis passing through the Sun's center and perpendicular to the plane of the ecliptic (or the plane of the solar equator). As in [Xue et al., 2005], we neglect the difference between the plane of the ecliptic and the plane of the solar equator. The longitude angle $\varphi_{0}$ is measured in the plane of the ecliptic from the central meridian counterclockwise: $\varphi_{0}=\left[0^{\circ}-360^{\circ}\right]$.

Let us note new approaches we use to calculate parameters of the ice-cream cone model. Unlike [Xue et al., 2005], in this paper: a) model CME parameters in 3D space are calculated separately for the CME body and its associated shock; b) not only velocities of these structures are calculated but also their positions along the model CME direction in 3D space at different instants; c) the boundary of the real CME body and shock projection onto the plane of the sky is determined not only by a discrete set of points in these structures, but also by approximating these structures by ellipses or ellipse segments.
At first glance, the ice-cream cone model is a very simple CME model and it is worthwhile to use more realistic CME models to solve the problem. Michalek [2006] adopted the CME cone model considered more realistic, with an elliptic shape of the base of the model cone. Thernisien et al. [2006] used an even more realistic CME model in the form of a magnetoplasma rope. One of the reasons for using the cone model proposed in [Xue et al., 2005] to calculate 3D parameters of CME and associated shock is the results obtained by Kim et al. [2011]. Kim et al. show that the CME radial velocities calculated with the CME models put forward by Michalek [2006] and Thernisien et al. [2006] are very close (the correlation coefficient is over 0.95) to the velocities calculated with the method worked out by Xue et al. [2005].

Our implementation of the method proposed by Gopalswamy and Yashiro [2011] is as follows:

1. For each HCME examined at different moments of its movement in the LASCO C3 field of view, we use the method from [Xue et al., 2005] to calculate the distance between the shock and the CME body $\Delta R$ along the model CME axis, as well as the radius of the CME body curvature boundary $R_{\mathrm{C}}$. Recall that, unlike [Xue et 
al., 2005], where 3D coordinates of the model CME were computed using a discrete set of points in the shock in the plane of the sky, in this paper we delineate both the shock and the CME body by ellipse segments (Figure 1).

2. From the relation [Russell, Mulligan, 2002]

$$
\Delta R / R_{C}=0.81\left[(\gamma-1) M^{2}+2\right] /\left[(\gamma+1)\left(M^{2}-1\right)\right]
$$

we find the Alfvén Mach number $M$ (it is assumed, as in [Kim et al., 2012] with the results of which we compare our results, that the adiabatic exponent $\gamma=4 / 3)$.

3. From the formula $M=\left(V_{\mathrm{SH}}-V_{\mathrm{SW}}\right) / V_{\mathrm{A}}$ we find the Alfvén velocity $V_{\mathrm{A}}$. Here $V_{\mathrm{SH}}$ is the shock velocity, $V_{\mathrm{SW}}$ is the velocity of the solar wind along which the shock propagates. As in [Gopalswamy, Yashiro, 2011], we suppose that a significant part of CME moves in the region of the slow SW whose velocity is found from [Sheeley et al., 1997]

$$
\left(V_{\mathrm{SW}}\right)^{2}=1.75 \cdot 10^{5}(1-\exp (-(R-4.5) / 15.2)) \text {. }
$$

4. From $V_{\mathrm{A}}=2.18 \cdot 10^{6} \mathrm{Bn}^{-1 / 2}$ [Gopalswamy, Yashiro, 2011] we determine the magnetic induction $B$ (in $G$ ). Here, $n$ is the particle density (in $\mathrm{cm}^{-3}$ ) in the coronal plasma, which is assumed to be equal to the electron density and is found from [Leblancet al., 1998]

$$
n(R)=3.3 \cdot 10^{5} R^{-2}+4.1 \cdot 10^{6} R^{-4}+8.0 \cdot 10^{7} R^{-6},
$$

where $R$ is the distance from the Sun's center to the observation point in solar radii.

\section{RESULTS}

Figure 1 shows HCME in the LASCO C3 field of view, with the CME body and shock boundaries delineated by ellipse segments. Note that these structures are not always completely delineated in latitude within $360^{\circ}$. We outline only those parts of the structures that can be reliably identified as an HCME body or as a shock. The analysis has shown that the difference in the
3D parameters calculated for the same events with incomplete and complete $\left(360^{\circ}\right)$ delineations is relatively small.

The diffuse region boundary in front of the CME body (or CME frontal structure) is considered to be the shock because the velocity of this boundary relative to the surrounding slow SW exceeds the Alfvén velocity (the idea was first formulated by Sheeley et al. [2000]) and in certain regions of this boundary on brightness scans along the directions perpendicular to the boundary there are brightness jumps with a spatial size $(1-2) \delta R$ (Figure $1, b$ ), where $\delta R \approx 0.125 R_{\mathrm{S}}$ is the LASCO C3 spatial resolution. Such shocks are referred to as collisionless [Artsimovich, Sagdeev, 1979] because their front width is much smaller than the mean (approximately equal) free path of protons and electrons in collisions with protons in the coronal plasma $L_{\mathrm{C}}\left(L_{\mathrm{C}} \approx(1-3) R_{\mathrm{S}}\right.$ in the LASCO C3 field of view). According to [Artsimovich, Sagdeev, 1979], the real width of the collisionless shock front with respect to the coronal plasma and magnetic field characteristics is much smaller than $\delta R$. As an example, we estimate the front width of a perpendicular (i.e. propagating perpendicular to the magnetic field) collisionless shock. In this case, the width of the shock front $\Delta_{\mathrm{SH}} \sim r_{\mathrm{i}}$, where $r_{\mathrm{i}}$ is the ion Larmor radius. Let us estimate $\Delta_{\mathrm{SH}}$ for $R=23.82 R_{\mathrm{S}}$. At this distance, according to our calculations, $B=6.39 \cdot 10^{-3} \mathrm{G}$. We make this estimate for protons as ion type predominant in the coronal plasma. A fairly reasonable estimate of proton temperature $T_{\mathrm{p}}$ at the selected distance is $T_{\mathrm{p}}=5 \cdot 10^{5} \mathrm{~K}$. Hence it follows that

$$
\begin{aligned}
& \Delta_{\mathrm{SH}} \sim r_{\mathrm{i}}=1.02\left(T_{\mathrm{p}} / 11605\right)^{1 / 2} / B=1.02\left(5 \cdot 10^{5} / 11605\right)^{1 / 2} / \\
& \left(6.39 \cdot 10^{-3}\right) \approx 1048 \mathrm{~m}<<\delta R \approx 0.125 R_{\mathrm{S}} \approx 87000000 \mathrm{~m} .
\end{aligned}
$$

For the events from Table 1, Table 2 lists the calculation results on the shock position $R / R_{\mathrm{S}}$ and velocity $V_{\mathrm{SH}}$ in $3 \mathrm{D}$ space at different instants, as well as the slow
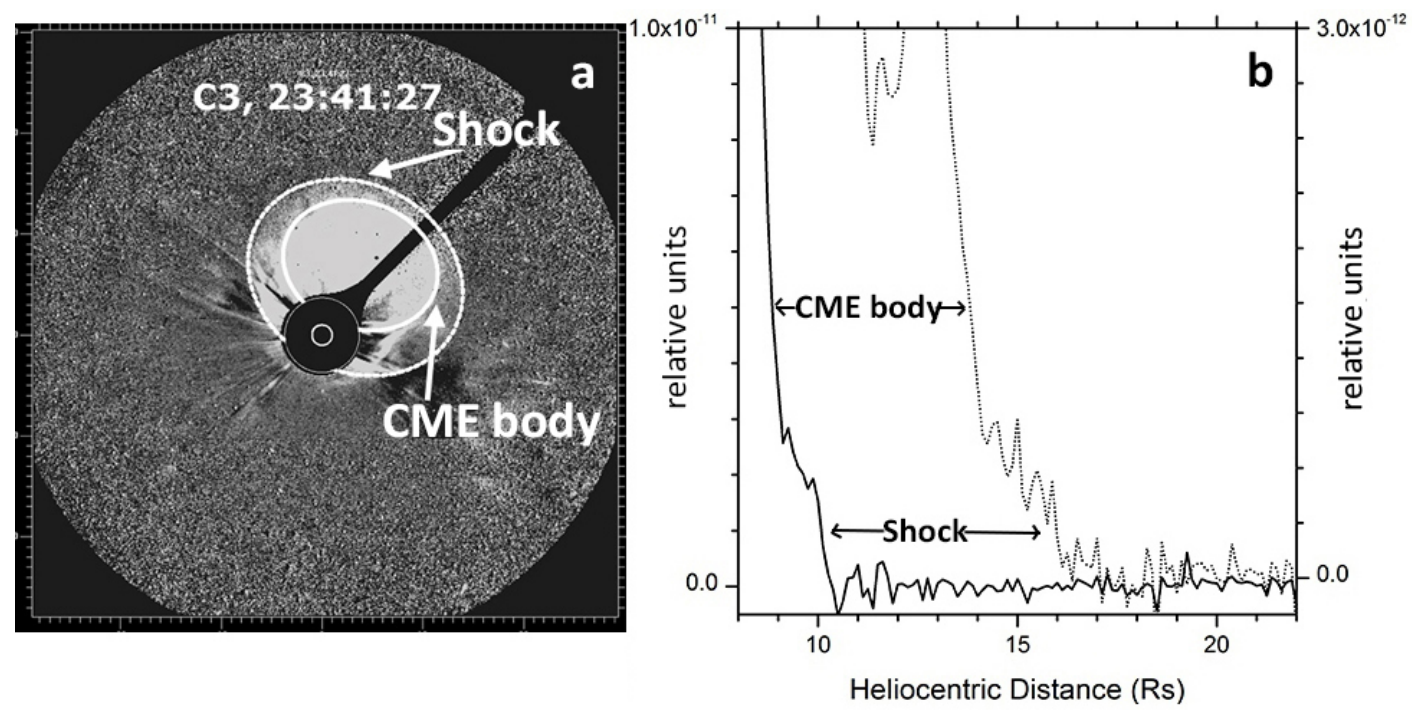

Figure 1. HCME recorded on January 15, 2005 at 23:41:27 (a). White ellipses indicate boundaries of the CME body (bright inner region) and of the shock (the boundary of the outer diffuse region). Brightness distributions along $30^{\circ} \mathrm{NW}$ for two instants $\left(t_{1}=23: 17: 25\right.$ and $\left.t_{2}=23: 41: 27\right)(b)$. Clearly defined are the brightness jumps indicated by the arrows from the word "shock" with a spatial size $(1-2) \delta R$, where $\delta R \approx 0.125 R_{\mathrm{S}}$ is the LASCO C3 spatial resolution. This shock is assumed to be collisionless. The data on the left vertical axis on panel $b$ are shown by the left curve; the data on the right vertical axis, by the right curve 
Table 2

\begin{tabular}{|c|c|c|c|c|c|c|}
\hline$R / R_{\mathrm{S}}$ & $V_{\text {sh }}$ & $V_{\mathrm{SW}}$ & $V_{\mathrm{A}}$ & $\Delta R / R_{\mathrm{C}}$ & $M$ & $B$ \\
\hline 9.3 & 2121.6 & 217.9 & 1018 & 0.4 & 1.9 & 0.031 \\
\hline 11.8 & 1646.9 & 258.4 & 1009.8 & 0.2 & 1.4 & 0.023 \\
\hline 13.4 & 1956.5 & 278.4 & 569.2 & 0.3 & 2.9 & 0.011 \\
\hline 13.8 & 2382.5 & 282.7 & 1528.3 & 1.1 & 1.4 & 0.03 \\
\hline 14.1 & 2079.9 & 286.5 & 448.4 & 0.2 & 4 & 0.009 \\
\hline 15.2 & 2997 & 297.1 & 1120.3 & 0.3 & 2.4 & 0.02 \\
\hline 15.2 & 1573.1 & 297.3 & 873.8 & 0.2 & 1.5 & 0.016 \\
\hline 16.4 & 1579.4 & 308.6 & 803.3 & 0.8 & 1.6 & 0.0131 \\
\hline 16.5 & 1830.7 & 309 & 951.6 & 0.8 & 1.6 & 0.016 \\
\hline 18.6 & 1992.6 & 325.1 & 620.8 & 0.2 & 2.7 & 0.009 \\
\hline 21 & 2205 & 340.6 & 1399.7 & 1.2 & 1.3 & 0.0178 \\
\hline 21.1 & 1534.7 & 340.9 & 735.1 & 0.7 & 1.6 & 0.009 \\
\hline 21.5 & 2990.3 & 343 & 969.7 & 0.2 & 2.7 & 0.012 \\
\hline 21.8 & 1604.4 & 345 & 858.5 & 0.8 & 1.5 & 0.011 \\
\hline 23.3 & 1817.3 & 352.5 & 739.1 & 0.6 & 2 & 0.008 \\
\hline 23.4 & 2135.9 & 352.8 & 934 & 0.4 & 1.9 & 0.011 \\
\hline 23.8 & 2082.1 & 354.8 & 570.8 & 0.2 & 3 & 0.006 \\
\hline 25.2 & 2554.8 & 360.7 & 963.2 & 0.6 & 2.3 & 0.01 \\
\hline 28.2 & 2050.3 & 371.8 & 1277.4 & 1.2 & 1.3 & 0.012 \\
\hline 29.7 & 1621.1 & 376.3 & 826.6 & 0.8 & 1.5 & 0.007 \\
\hline 30.5 & 1804.4 & 378.7 & 556.7 & 0.4 & 2.6 & 0.005 \\
\hline 31.7 & 2141.4 & 381.8 & 854.2 & 0.4 & 2.1 & 0.007 \\
\hline 33.5 & 2816.4 & 386.1 & 1048.5 & 0.3 & 2.3 & 0.008 \\
\hline 34.6 & 2558.3 & 388.3 & 805.2 & 0.3 & 2.7 & 0.006 \\
\hline 42.7 & 2455.9 & 401 & 514.9 & 0.2 & 4 & 0.003 \\
\hline 43.6 & 2619.4 & 402 & 861.8 & 0.3 & 2.6 & 0.005 \\
\hline
\end{tabular}

SW velocity $V_{\mathrm{SW}}$, Alfvén velocity $V_{\mathrm{A}}$, parameter $\Delta R / R_{\mathrm{C}}$, Alfvén Mach number $M$, and magnetic field values $B$ (without specifying the events to which each parameter refers). The parameters from this Table are used for Figures 2-4.

Figure 2 presents the magnetic field calculation results obtained from HCME 3D parameter calculations. This Figure indicates that the obtained dependence $B(R)$ (white circles) agrees quite well with the expected values of the magnetic field in the slow SW (the solid line in Figure 2, $a$ ). This line is a function graph

$$
B_{\mathrm{R}}=11.1 \cdot 10^{-5}\left(215.5 /\left(R / R_{\mathrm{S}}\right)\right)^{2}[\mathrm{G}],
$$

where $11.1 \cdot 10^{-5}$ is $B_{R}$ in the horizontal (parallel to the plane of the solar equator) part of the slow SW in Earth's orbit; 215.5 is the distance from the Sun to Earth's orbit in solar radii. It can be argued that in both fast and slow SW, the magnetic field radial component $B_{\mathrm{R}}$ starting at $R \approx 3 R_{\mathrm{S}}$ varies in inverse proportion to the squared distance (for interplanetary space (see Figure 4.1 on page 186 in [Schwenn, Marsch, 1990]). However, we should make a proviso for the slow SW. For relatively large distances from the Sun, including those near
Earth's orbit, this is true only for horizontal (i.e. parallel to the plane of the solar equator) parts of the slow SW. The reason for this is the existence of interaction between fast and slow SW streams if a part of the slow SW is at a relatively large angle to the plane of the solar equator [Schwenn, Marsch, 1990; Fainshtein, 1991]. This interaction leads to a significant change in the magnetic field in the vicinity of the inclined parts of the slow SW as compared to its horizontal parts. Therefore, if we want to estimate $B_{\mathrm{R}}$ in the corona from values of this field component in Earth's orbit, we should use the $B_{\mathrm{R}}$ values in Earth's orbit only in horizontal parts of the slow SW. This question has been discussed in detail in [Fainshtein, 1991], where characteristic values of the magnetic field and proton density in some horizontal parts of the slow SW in Earth's orbit are given.

It is easy to see that in the range of the same distances $(9-15) R_{\mathrm{S}}$, for which the magnetic field is calculated both in our paper and in [Kim et al., 2012], the difference in the results is relatively small: the average field value $\langle B\rangle=0.02 \mathrm{G}$ we derived for the said period, in [Kim et al., 2012] $<B>=0.019$ and $0.015 \mathrm{G}$. 
Here, the first value of $\langle B>$ refers to the field calculations performed using both $\Delta R / R_{\mathrm{C}}$ and $\rho_{\mathrm{d}} / \rho_{\mathrm{u}}$ (density jump in the shock front, where $\rho_{\mathrm{d}}$ is the plasma density behind the shock; $\rho_{u}$, in front of the shock [Kim et al., 2012]), to find the Alfvén Mach number $M$. The second mean-field value refers to the field calculations using only $\Delta R / R_{\mathrm{C}}$ to find $M$, as is done in our paper. The difference between the mean magnetic field values obtained in [Kim et al., 2012] is largely due to the use of the simplified CME model in 3D space in this paper, which includes such simplifying assumptions as CME radial motion, constancy of the angular size of CME when in motion, spherical shape of the outer boundary of the CME body and associated shock, etc. Let us note here that this difference is within the measurement accuracy with which the method proposed by Gopolswamy, Yashiro, [2011] allows us to determine the magnetic field strength in the corona. The accuracy in determining $B$ is affected by many factors such as the approximate velocity of the slow SW, the relations that allow us to determine $M$ ( $M$ refers to $\Delta R / R_{\mathrm{C}}$ or to plasma density jump in the shock front $\rho_{d} / \rho_{u}$ ), the use of the same distance dependence of plasma density for all events, etc. For example, Figure 2, $b$ suggests that the magnetic field values calculated using the relationship of the Mach number with $\Delta R / R_{\mathrm{C}}$ are much smaller than $B(R)$, obtained using the $M$ dependence on $\rho_{\mathrm{d}} / \rho_{\mathrm{u}}$.

Figure 3, $a$ shows the spread of $V_{\mathrm{A}}$ values derived from HCME observations; Figure $3, b$, by comparison, shows the $V_{\mathrm{A}}$ dependence on the shock position obtained in [Kim et al., 2012]. Figure 3, $c$ depicts $V_{\mathrm{A}}$ as a function of distance, calculated for the slow (bottom band) and fast SW (top band), using magnetic field and proton density values in Earth's orbit and their distance dependence. We assume that within $5 R_{\mathrm{S}}-1$ AU (distance from the Sun to Earth's orbit), the magnetic field radial component $B_{\mathrm{R}} \sim 1 / R^{2}$ in both the fast and slow $\mathrm{SW}$, the proton density $n_{\mathrm{p}} \sim 1 / R^{2}$ in the fast SW, whereas in the slow SW it varies with distance according to the law obtained for electron density variations $n_{\mathrm{e}}(R)$ in [Leblanc et al., 1998].

Details of plotting of $V_{\mathrm{A}}(R)$ in Figure 3 are given in [Fainshtein, Egorov, 2017]. In Figure 3, $c$, the dependences are compared with the spread of points in Figure $3, a, b$, because the dependences in Figure $3, c$, are, from our point of view, the most accurate estimates of $V_{\mathrm{A}}(R)$ on record. Figure 3 indicates that within $(9-15) R_{\mathrm{S}}$ the mean Alfvén velocity $\left\langle V_{\mathrm{A}}\right\rangle$ we obtained from HCME observations is $938 \mathrm{~km} / \mathrm{s}$, and according to the data from [Kim et al., 2012] $\left\langle V_{\mathrm{A}}\right\rangle=709 \mathrm{~km} / \mathrm{s}$ (559 $\mathrm{km} / \mathrm{s}$ ). At the same time, the plot drawn from the HCME data more clearly demonstrates the Alfvén velocity decrement with distance. Note also that the spread of the $V_{\mathrm{A}}$ values for each shock wave position within up to $15 R_{\mathrm{S}}$, obtained both in our calculations and in [Kim et al., 2012], is much larger than that derived in Alfvén velocity calculations from magnetic field and plasma density measurements in Earth's orbit (Figure 3, c). We assume that this indicates a lower accuracy of Alfvén velocity calculations with the methods proposed in [Gopalswamy, Yashiro, 2011; Kim et al., 2012].
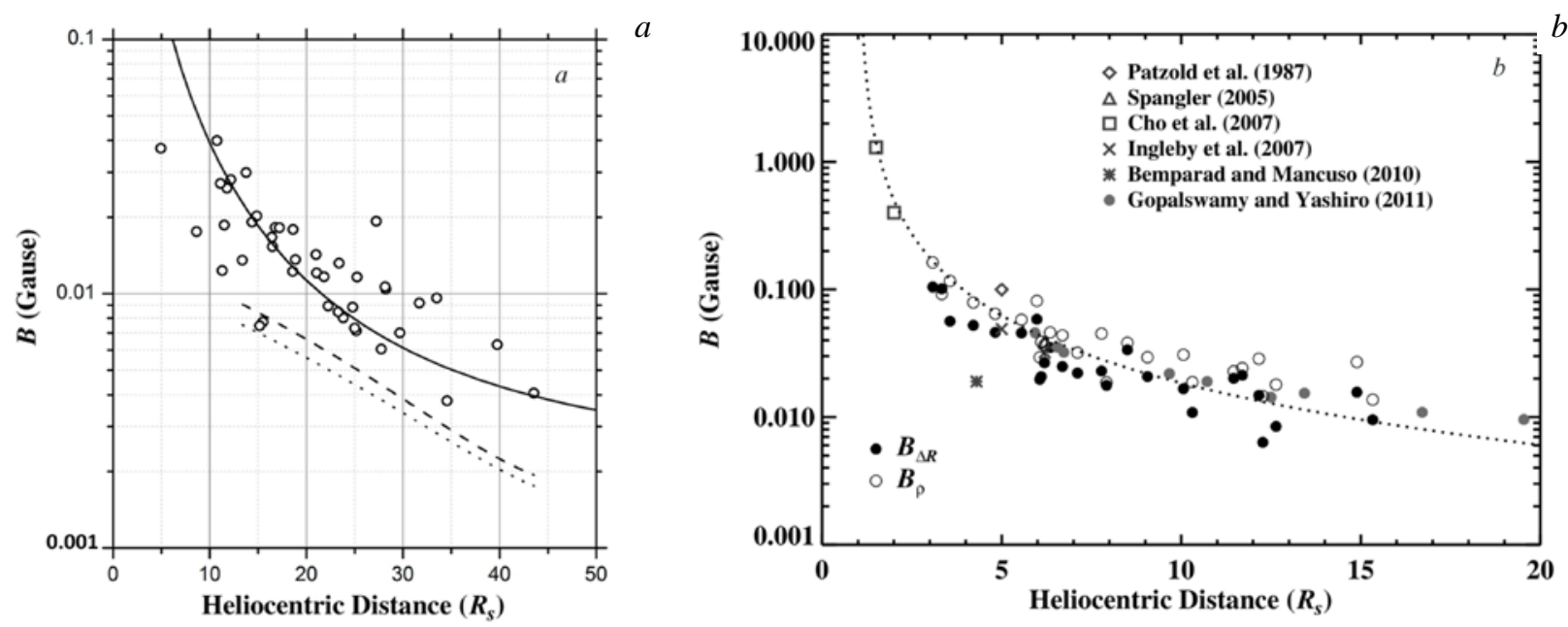

Figure 2. Panel $a$ : magnetic field values $B(R)$ obtained by calculating HCME 3D parameters at different instants for each event under study (circles). The solid line $\left(B_{\mathrm{R}}=11.1 \cdot 10^{-5}\left(215.5 /\left(R / R_{\mathrm{S}}\right)\right)^{2} \mathrm{G}\right)$ is the magnetic field radial component in a horizontal part of the slow SW as a function of distance (for more detail, see the description of Figure 2 in the text) Two lines below are field calculations under the assumption that the CME paraxial region moves in the fast $\mathrm{SW}$ at $V_{\mathrm{SW}}=600 \mathrm{~km} / \mathrm{s}$ (dashes) and $800 \mathrm{~km} / \mathrm{s}$ (dotted line) regardless of the distance, and with the distance-dependent proton density and magnetic field radial component typical for the fast SW: $n_{\mathrm{p}}=3\left(215.5 /\left(R / R_{\mathrm{S}}\right)\right)^{2} \mathrm{~cm}^{-3}, B_{\mathrm{R}}=3 \cdot 10^{-5}\left(215.5 /\left(R / R_{\mathrm{S}}\right)\right)^{2} \mathrm{G}$. Only regression lines are shown here. Panel $b$ : Figure 8 from [Kim et al., 2012] (@ AAS. Reproduced with permission): black circles indicate $B(R)$ values obtained from the relation associating the Mach number with $\Delta R / R_{\mathrm{C}}$; white circles, with density in the shock front. Other symbols show magnetic induction values obtained by different authors with other methods (see [Kim et al., 2012] and references therein) 

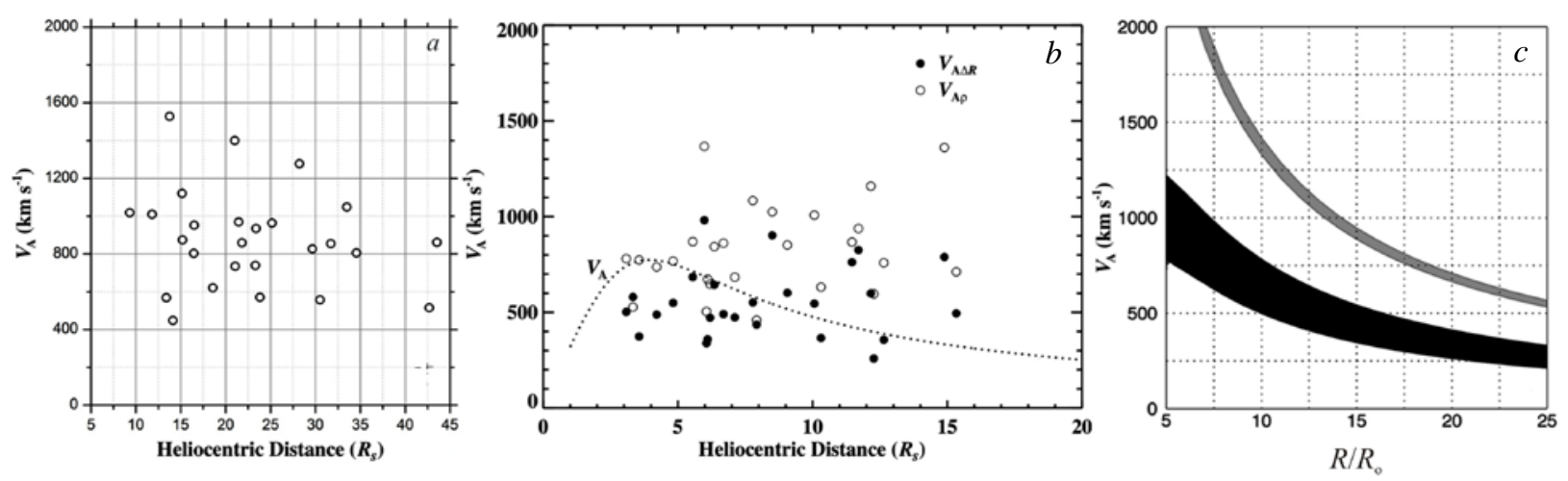

Figure 3. Panel $a$ : distance-dependent spread of Alfvén velocity $V_{\mathrm{A}}$ values (circles) obtained from HCME data in 3D space. Panel $b$ : Figure 7 from [Kim et al., 2012] (C AAS. Reproduced with permission): the dotted line is the $V_{\mathrm{A}}(R)$ dependence from [Mann et al., 1999]; black circles are the $B(R)$ values obtained from the relation associating the Mach number with $\Delta R / R_{C}$; white circles, with density in the shock front. Panel $c$ : the $V_{\mathrm{A}}(R)$ dependence obtained for the slow (black band) and fast SW (gray band) from magnetic field and proton density values in Earth's orbit and certain dependences of their variation with distance (see [Fainshtein, Egorov, 2017])

Figure 4, $a$, shows the spread of our Alfvén Mach numbers $M$ for various shock positions. Figure $4, b$ presents a similar result from [Kim et al., 2012]. It can be seen that within (9-15) $R_{\mathrm{S}}$, the spread of values and the average Mach number $M$ for the calculations of two types are close: $\langle M>=2.21$ according to our calculations and $<M>=2.03$ (2.55) according to the results received in [Kim et al., 2012]. Recall that the value of 2.55 is derived by averaging $M$, obtained using only parameter $\Delta R / R_{\mathrm{C}}$; and the value of 2.03 , using both $\Delta R / R_{\mathrm{C}}$ and the plasma density jump $\rho_{\mathrm{d}} / \rho_{\mathrm{u}}$ at the shock front .

Figure 4, $c$ illustrates the $\Delta R / R_{\mathrm{C}}$ spread we obtained for different shock positions; Figure $4, d$, the spread of the same parameter from [Kim et al., 2012]. Within (9-15) $R_{\mathrm{S}}$, according to our calculations, $\left\langle\Delta R / R_{\mathrm{C}}>=0.39\right.$; according to [Kim et al., 2012], $<\Delta R / R_{\mathrm{C}}>=0.31$.
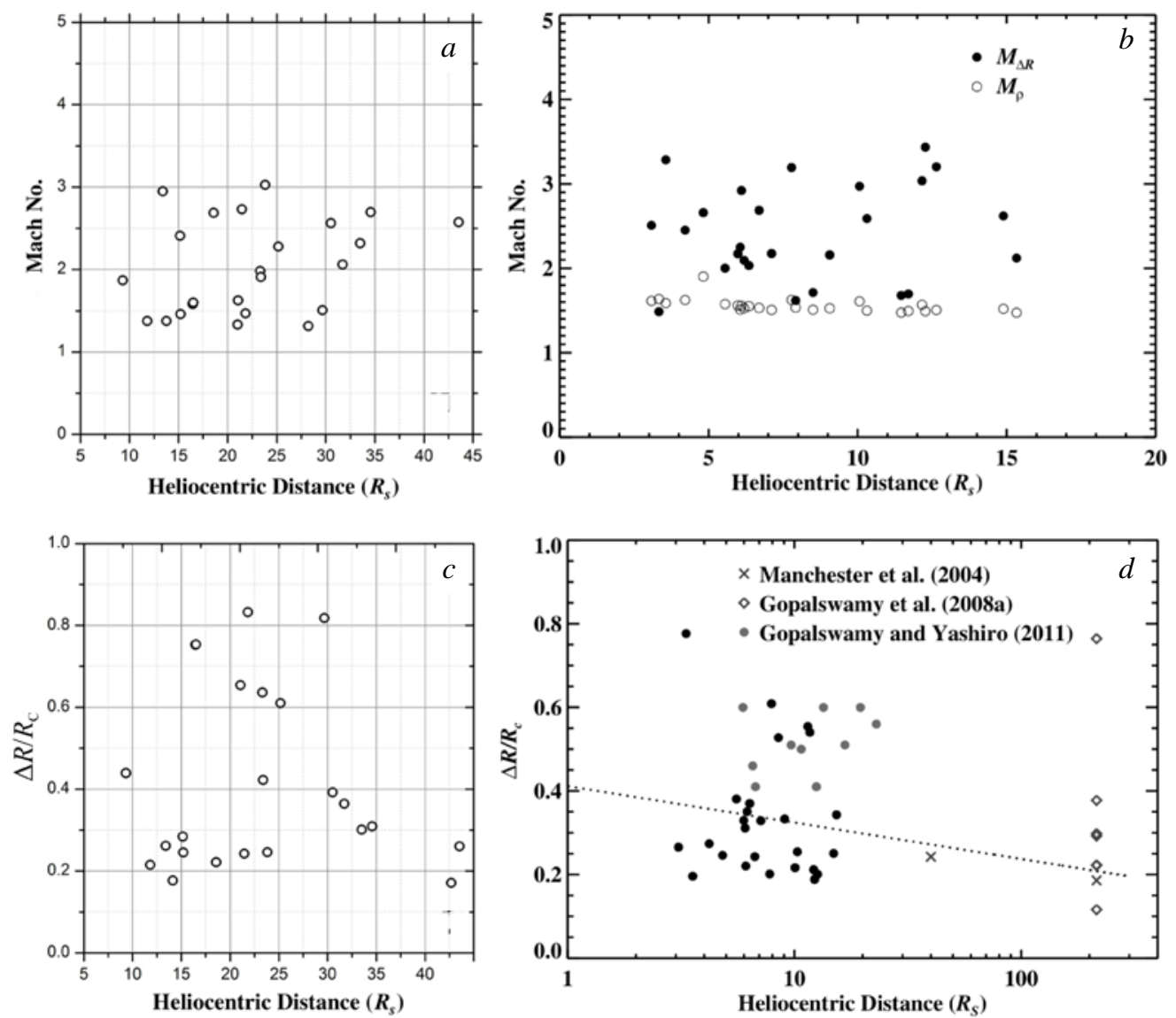

Figure 4. Panel $a$ : spread of the Mach numbers we derived for various shock positions. Panel $b$ : Figure 6 from [Kim et al., 2012] (C AAS. Reproduced with permission). Panel $c$ : spread of the $\Delta R / R_{\mathrm{C}}$ values we obtained; panel $d$ : Figure 2 from [Kim et al., 2012] (C AAS. Reproduced with permission) 


\section{EFFECT OF SOLAR WIND VELOCITY ON MAGNETIC FIELD CALCULATION}

As in [Gopalswamy, Yashiro, 2011], when calculating the magnetic field strength, we implicitly assume that the paraxial regions of all CMEs, whose properties are used to find the magnetic field strength, move in the slow SW. That is why we use the distance-dependent velocity of the slow SW from [Sheeley et al., 1997] as the velocity of SW in which a shock propagates. In fact, for many CMEs it is rather difficult to determine which parts of the CMEs move in the slow SW. Moreover, in some cases, the entire CME can move in the fast SW in the LASCO C3 field of view. At sufficiently high velocities (for example, higher than $1500 \mathrm{~km} / \mathrm{s}$ ), there may be a shock ahead of it. Assuming that in all the events considered CME moves in the fast SW, we find the magnetic field radial distribution by the method from [Gopalswamy, Yashiro, 2011]. The regression lines for the dependence of the magnetic induction on the shock position are shown in Figure 2. It is seen that the magnetic field here is much smaller than that in the slow SW.

Thus, the calculations of magnetic field radial distributions by the method from [Gopalswamy, Yashiro, 2011] should be preceded either by finding out which CME region is moving in the slow SW and which one in the fast SW or by proof that the entire CME is moving in the fast SW. We plan to use magnetic field calculations in the potential approximation and white corona images from LASCO C2, C3 data to propose a possible solution to this problem in our next paper. In particular, as a separate group we will analyze CMEs whose central part moves along a streamer belt part approximately perpendicular to the plane of the sky, or, which is the same, parallel to the plane of the solar equator (such parts of the streamer belt or slow SW will be called, as in this paper, horizontal parts). The streamer belt parts of this type are observed in the coronagraph field of view as bright rays, and in this case it is easy to determine the CME part that moves in the slow SW. We also propose to adopt one more method for isolating limb CMEs whose central part moves along the streamer, i.e. in the slow SW. It is known that the spatial configuration of the coronal streamer belt closely coincides with the configuration of the magnetic field neutral line (NL) on the source surface, which is obtained from the field calculations in the potential approximation [Schwenn, Marsch, 1990]. The radius of the source surface usually varies in field calculations within (1.6-3.25) $R_{\mathrm{S}}$. Hence it follows that to the streamer belt parts roughly perpendicular to the plane of the sky correspond the NL segments roughly parallel to the plane of the equator. If at the moment of CME observation in the LASCO C2 field of view in the plane of the sky there is a middle NL segment approximately parallel to the plane of the equator and the CME axis is close in latitude to the latitude of this section, we can be sure that a significant part of the CME is moving in the slow SW.

Figure 5 shows the neutral line calculated in WSO [http://wso.stanford.edu/synsourcel.html] with areas located at small angles to the plane of the solar equator (i.e. almost parallel to this plane), and CME moving along a bright streamer corresponding to one of such areas, and hence its central part is moving in the slow SW. Note that this approach for isolating CMEs, most of which move in the slow SW, can also be used for CMEs propagating in directions close to the Sun-Earth axis. In this case, using, for example, the ice-cream cone model for CME, we find the direction of the model CME and compare the latitude of this direction with the latitude of the horizontal NL segment calculated in the potential approximation on the source surface on the day of the CME observation (if such a segment exists!). If the latitude of the CME direction and the latitude of the horizontal NL segment are close, all the conclusions that we have drawn for the limb event in Figure 5 are also valid in this case: the most part of this CME moves in the slow SW.

\section{CONCLUSIONS}

1. Using the method proposed by Gopalswamy and, Yashiro [2011] and then elaborated by Kim et al. [2012], we have obtained the magnetic field spatial distribution $B(R)$ approximately along the Sun-Earth axis. To do this, we used data on halo CMEs (HCMEs) whose sources were largely located near the center of the solar disk.

2. The HCME parameters necessary for calculating the magnetic field strength in 3D space were found using the ice-cream cone model for CME [Xue et al., 2005].

3. We adopted new approaches to calculate the parameters of the ice-cream cone model. Unlike [Xue et al., 2005], in this paper: a) the parameters of the model CME in 3D space were calculated separately for the CME body and associated shock; b) not only velocities of these structures were calculated but also their positions along the model CME direction in 3D space at different instants; c) the boundary of the projection of the CME body and shock onto the plane of the sky was distinguished not only by a discrete set of points in these structures, but also by the approximation of these structures by ellipses or ellipse segments.

4. Through the field calculations, we managed to double the maximum distance as compared to that in [Gopalswamy, Yashiro, 2011; Kim et al., 2012], for which we determined the magnetic field distribution $B(R)$ : approximately from $21 R_{\mathrm{S}}$ to $43 R_{\mathrm{S}}$.

5. It has been shown that the $B(R)$ distribution we obtained is similar on average to the distance dependence of the field radial component varying according to the law $B_{\mathrm{R}}=B_{\mathrm{RE}}\left(215.5 /\left(R / R_{\mathrm{S}}\right)\right)^{2}$, where $B_{\mathrm{RE}}=11 \cdot 10^{-5} \mathrm{G}$ is a possible value of $B_{R}$ in the horizontal part of the slow SW in Earth's orbit (see [Fainshtein, 1991] for horizontal parts of the slow SW).

6 . The average values of the magnetic field $B$, Alfvén velocity $V_{\mathrm{A}}$, Alfvén Mach number $M$, and parameter $\Delta R / R_{\mathrm{C}}$ obtained in this paper and in [Kim et al., 2012] within (9-15) $R_{\mathrm{S}}$ proved to be relatively close (with a difference within $\sim 5-26 \%$ ). We compared the calculated $B, V_{\mathrm{A}}$, and $M$ with the total results [Kim et al., 2012], obtained using the relations between the Mach number and both the $\Delta R / R_{\mathrm{C}}$ and $\rho_{\mathrm{d}} / \rho_{\mathrm{u}}$ parameters. 


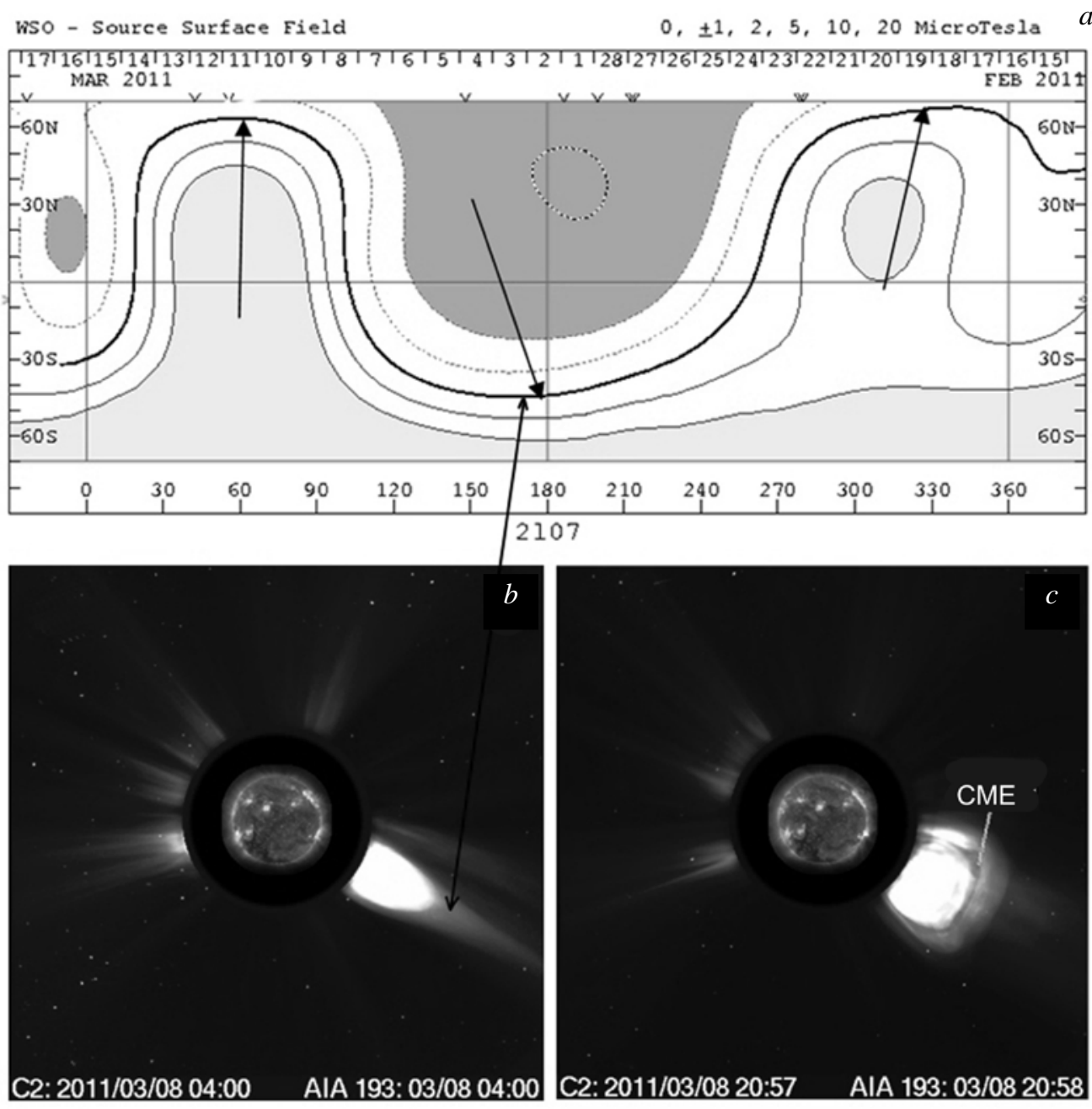

Figure 5. Panel $a$ : magnetic field distribution over the source surface $\left(2.5 R_{\mathrm{S}}\right)$ from the field calculations in the potential approximation in WSO [http://wso.stanford.edu/synoptic/WSO-S.2107.gif]. Arrows indicate NL segments, located at small angles to the plane of the solar equator. The CME impact on the streamer led to a decrease in its brightness ahead of the CME. LASCO C2 white corona images $(b, c)$ : the coronal streamer before CME occurrence (indicated by an arrow) and streamer-related NL segment $(b)$; CME the middle part of which moves along the coronal streamer shown on panel $b(c)$

7. The $B(R)$ distributions found by the method we proposed along a direction close to the Sun-Earth axis can be used: a) to estimate the magnetic field in Earth's orbit from the solar data for some characteristic intervals (for example, for different phases of solar activity); b) to compare them with radial field distributions obtained from MHD calculations.

Finally, we note that the quite satisfactory agreement between our magnetic field calculations and the calculations made in [Kim et al., 2012] is an additional reason to believe that the ice-cream cone model for CME makes it possible to adequately identify both CME characteristics and characteristics of its motion in 3D space.

8. The $V_{\mathrm{A}}(R)$ radial distributions we and Kim et al. [2012] obtained have been compared with the radial dependence of the Alfvén velocity in the slow SW from [Fainshtein, Egorov, 2017], which the authors consider the most accurate (within errors in determining $V_{\mathrm{A}}(R)$ ) of the known dependences (for example, as compared to the popular $V_{\mathrm{A}}(R)$ dependence from [Mann et al., 1999]). We have concluded that the largest of $V_{\mathrm{A}}$ values, found both in this paper and in [Kim et al., 2012], are overestimated as compared to the real Alfvén velocity values.

9. We have put forward a method for isolating CMEs a significant part of which moves in the slow SW.

We are grateful to the LASCO team for the free access to coronagraph data. The study was carried out under the ISTP SB RAS 2016-2018 R\&D Plan II.16.1.6., entitled "Geoeffective Processes in the Sun's Chromosphere and Corona” (basic project), and with partial support of RFBR grants Nos 15-02-01077-a and 16-32-00315. The work was done as part of Goszadanie 2018, project No. 00700163-18-00 of 12.01.2018.

\section{REFERENCES}

Artsimovich L.A., Sagdeev R.Z. Fizika plazmy dlya fizikov [Plasma physics for physicists]. Moscow, Atomizdat. 1979, 320 p. (In Russian).

Bemporad A., Mancuso S. First complete determination of plasma physical parameters across a coronal mass ejectiondriven shock. Astrophys. J. 2010, vol. 720, pp. 130-143. DOI: 10.1088/0004-637X/720/1/130. 
Brueckner G.E., Howard R.A., Koomen M.J., Korendyke C.M., Michels D.J., Moses J.D. The Large Angle Spectroscopic Coronagraph (LASCO). Solar Phys. 1995, vol. 162, pp. 357-402. DOI: 10.1007/BF00733434.

Bogod V.M., Yasnov L.V. Determination of the structure of the coronal magnetic field using microwave polarization measurements. Solar Phys. 2016, vol. 291, pp. 3317-3328.

Cho K.-S., Lee J., Gary D.E., Moon Y.-J., Park Y.D. Magnetic field strength in the solar corona from type II band splitting. Astrophys. J. 2007, vol. 665, p. 799.

Fainshtein V.G. The interaction effect of fast and slow solar wind streams in interplanetary space on wind characteristics at the Earth's orbit. Solar Phys. 1991, vol. 136, pp. 169189. DOI: $10.1007 / \mathrm{BF} 00151702$.

Fainshtein V.G. Method for determining the parameters of full halo coronal mass ejections. Geomagnetism and Aeronomy. 2006, vol. 46, iss. 3, pp. 339-349. DOI: 10.1134/S001679320603008X.

Fainshtein V.G., Egorov Ya.I. Origin of a CME-related shock within the LASCO C3 field-of-view. Eprint arXiv.2017. arXiv:1712.09051.

Gopalswamy N., Akiyama S., Yashiro S., Michalek G., Lepping R.P. Solar sources and geospace consequences of interplanetary magnetic clouds observed during solar cycle 23 . J. Atmos. Solar-Terr. Phys. 2008, vol. 70, p. 245.

Gopalswamy N., Yashiro S. The strength and radial profile of the coronal magnetic field from the standoff distance of a coronal mass ejection-driven shock. Astrophys. J. Lett. 2011, vol. 736, article id. L17, 5 p. DOI: 10.1088/20418205/736/1/L17.

Howard R.A., Moses J.D., Vourlidas A., et al. Sun Earth Connection Coronal and Heliospheric Investigation (SECCHI). Space Sci. Rev. 2008, vol. 136, iss. 1-4. pp. 67115. DOI: $10.1007 / \mathrm{s} 11214-008-9341-4$.

Ingleby L.D., Spangler S.R., Whiting C.A. Probing the large-scale plasma structure of the solar corona with Faraday rotation measurements. Astrophys. J. 2007, vol. 668, p. 520.

Kaiser M.L., Kucera T.A., Davila J.M., St. Cyr O.C., Guhathakurta M., Christian E. The STEREO mission: an introduction. Space Sci. Rev. 2008, vol. 136, pp. 5-16. DOI: 10.1007/s11214-007-9277-0.

Kim T., Moon Y., Na H. Comparison of CME radial velocities from a flux rope model and an ice cream cone model. American Geophysical Union, Fall Meeting 2011, abstract \#SH51A-1992.

Kim R.-S., Gopalswamy N., Moon Y.-J., Cho K.-S., Yashiro S. Magnetic field strength in the upper solar corona using white-light shock structures surrounding coronal mass ejections. Astrophys. J. 2012, vol. 746, article id. 118, 8 p. DOI: $10.1088 / 0004-637 X / 746 / 2 / 118$.

Landau L.D., Lifshitz E.M. Gidrodinamika [Fluid mechanics]. 1988, 733 p. (In Russian).

Leblanc Y., Dulk G.A., Bougeret J.-L. Tracing the electron density from the corona to 1 au. Solar Phys. 1998, vol. 183, pp. 165-180. DOI: 10.1023/A:1005049730506.

Lee J. Radio emissions from solar active regions. Space Sci. Rev. 2007, vol. 133, pp. 73-102. DOI: 10.1007/s11214007-9206-2.

Lin H., Penn M.J., Tomczyk S. A new precise measurement of the coronal magnetic field strength. Astrophys. $J$. 2000, vol. 541, pp. L83-L86. DOI: 10.1086/312900.

Manchester W.B., Gombosi T.I., Roussev I., Ridley A., de Zeeuw D.L., Sokolov I.V., Powell K.G., Tóth G. Modeling a space weather event from the Sun to the Earth: CME generation and interplanetary propagation. J. Geophys. Res.: Space Phys. 2004, vol. 109, A02107.

Mann G., Klassen A., Estel C., Thompson B.J. Coronal transient waves and coronal shock waves. $8^{\text {th }}$ SOHO Work- shop "Plasma Dynamics and Diagnostics in the Solar Transition Region and Corona”: Proc. Paris, 1999, pp. 477-481.

Michalek G. An asymmetric cone model for halo coronal mass ejections. Solar Phys. 2006, vol 237, iss. 1, pp. 101-118. DOI: $10.1007 / \mathrm{s} 11207-006-0075-8$.

Pätzold M., Bird M.K., Volland H., Levy G.S., Seidel B.L., Stelzried C.T. The mean coronal magnetic field determined from HELIOS Faraday rotation measurements. Solar Phys. 1987, vol. 109, pp. 9-105. DOI: 10.1007/BF00167401.

Poomvises W., Gopalswamy N., Yashiro S., Kwon R.-Y., Olmedo O. Determination of the heliospheric radial magnetic field from the standoff distance of a CME-driven shock observable by the STEREO spacecraft. Astrophys. J. 2012, vol. 758, article id. 118, 6 p. DOI: 10.1088/0004-637X/758/2/118.

Rudenko G.V. Extrapolation of the solar magnetic field within the potential-field approximation from full-disk magnetograms. Solar Phys. 2001, vol. 198, pp. 5-30. DOI: 10.1023/A:1005270431628.

Rudenko G.V., Myshyakov I.I. Analysis of reconstruction methods for nonlinear force-free fields. Solar Phys. 2009, vol. 257, pp. 287-304. DOI: 10.1007/s11207-009-9389-7.

Russell C.T., Mulligan T. On the magnetosheath thicknesses of interplanetary coronal mass ejections. Planet. Space Sci. 2002, vol. 50, pp. 527-534. DOI: 10.1016/S00320633(02)00031-4.

Sahal-Bréchot S., Malinovsky M., Bommier V. The polarization of the O VI 1032 A. Astron. Astrophys. 1986, vol. 168, pp. 284-300.

Sheeley N.R., Jr., Wang Y.-M., Hawley S.H., et al. Measurements of flow speeds in the corona between 2 and $30 R_{0}$. Astrophys. J. 1997, vol. 484, pp. 472-478. DOI: $10.1086 / 304338$.

Sheeley N.R., Hakala W.N., Wang Y.-M. Detection of coronal mass ejection associated shock waves in the outer corona. J. Geophys. Res. 2000, vol. 105, pp. 5081-5092. DOI: 10.1029/1999JA000338.

Schmidt J.M., Cairns I.H., Gopalswamy N., Yashiro S. Coronal magnetic field profiles from shock-CME standoff distances. $J$. Geophys. Res.: Space Phys. 2016, vol. 121, pp. 9299-9315. DOI: 10.1002/2016JA022956.

Schwenn R., Marsch E. (Eds.) Physics of the Inner Heliosphere I. Large-Scale Phenomena. Springer-Verlag, 1990, 293 p. DOI: 10.1007/978-3-642-75361-9.

Spangler S.R. The strength and structure of the coronal magnetic field. Space Sci. Rev. 2005, vol. 121, pp. 189-200. DOI: $10.1007 / \mathrm{s} 11214-006-4719-7$.

Thernisien A.F.R., Howard R.A., Vourlidas A. Modeling of flux rope coronal mass ejections. Astrophys. J. 2006, vol. 652, pp. 763-773. DOI: $10.1086 / 508254$.

Wiegelmann T. Nonlinear force-free modeling of the solar coronal magnetic field. J. Geophys. Res. 2008, vol. 113, A03S02. DOI: 10.1029/2007JA012432.

Xue X.H., Wang C.B., Dou X.K. An ice-cream cone model for coronal mass ejections. J. Geophys. Res.: Space Phys. 2005, vol. 110, iss. A8, CiteID A08103. DOI: 10.1029/2004JA010698.

URL: https://cdaw.gsfc.nasa.gov/CME_list/HALO/halo.html (accessed September 16, 2017).

URL: http://wso.stanford.edu/synsourcel.html (accessed September 16, 2017).

URL: http://wso.stanford.edu/synoptic/WSO-S.2107.gif (accessed September 16, 2017).

How to cite this article

Fainstein V.G., Yegorov Ya.I. Radial distributions of magnetic field strength in the solar corona as derived from data on fast halo CMEs Solar-Terrestrial Physics. 2018, vol. 4, no. 1, pp. 3-11. DOI: 10.12737/stp-41201801. 